INVESTIGACIÓN/RESEARCH

\title{
RELACIÓN ENTRE EL NÚMERO DE ACCIDENTES LABORALES Y LA Q DE CALIDAD EN ESTABLECIMIENTOS HOTELEROS DE ANDALUCÍA
}

\author{
Mercedes Raquel García Revilla \\ Universidad a Distancia de Madrid (UDIMA), Madrid, España \\ mercedesraquel.garcia@udima.es
}

\section{RESUMEN}

Examinando las bases de datos disponibles y las organizaciones turísticas existentes se puede concluir que aparecen estudios realizados sobre accidentes laborales y su relación con otras variables. Pero en nuestro caso queremos valorar dicha relación en cuanto a establecimientos hoteleros con la certificación de calidad.

La Seguridad Industrial estudia esta siniestralidad analizando sus causas (por qué ocurren), sus fuentes (actividades comprometidas), sus agentes (medios de trabajo participantes), su tipo (cómo se producen o se desarrollan los hechos), todo ello con el fin de desarrollar la prevención.

En nuestro caso, como los accidentes ocurren porque la gente comete actos incorrectos o porque los equipos, herramientas, maquinarias o lugares de trabajo no se encuentran en condiciones adecuadas, el punto de vista preventivo lo analizamos a través de la incidencia en la siniestralidad laboral del subsector hotelero al obtener la $\mathrm{Q}$ de calidad turística, ya que ésta combina requisitos de sistema y de producto ${ }^{1}$. El accidente se produce porque existe el riesgo, por lo que un control adecuado del mismo lo evitaría a pesar de factores individuales.

El absentismo laboral o ausencia al trabajo fue tenido en cuenta por primera vez por Dubois en 1977, ya que se dio cuenta del tiempo de trabajo perdido en el siglo XIX debido a las largas jornadas de trabajo, en las que se incluían los días de fiesta.

La motivación y la satisfacción laboral han sido los elementos que han tenido más importancia en el fenómeno para la psicología social, analizados por Nicholson, Brown y Chadwick Jones. De manera general, califican la ausencia en el trabajo como:

\footnotetext{
${ }^{1}$ Las primeras certificaciones de calidad “Q" se concedieron en el año 1997 (Martín, 2014).
} 
- Un acto individual en el que se pueden elegir actividades alternas y un acto voluntario que comporta la resistencia individual y grupal con respecto a un sistema inflexible.

Cuestiones claves que nos surgen son:

- ¿Qué estructura organizativa debe tener la empresa para cumplir sus objetivos en cuanto a la prevención de riesgos laborales?

- ¿Cómo se pueden lograr sus objetivos en cuanto a prevención de riesgos se refiere?

- ¿Están las organizaciones actuales protegiendo adecuadamente a sus trabajadores?

- ¿Qué relación existe entre accidentes laborales y la certificación Q de calidad?

PALABRAS CLAVE: Accidentes de trabajo - Establecimientos - Hoteles - Q calidad Ventaja competitiva.

\title{
RELATIONSHIP BETWEEN THE NUMBER OF ACCIDENTS AND Q QUALITY HOTEL ESTABLISHMENTS OF ANDALUCIAN
}

\begin{abstract}
Examining the available databases and existing tourism organizations can conclude that appear studies on accidents and their relationship with other variables. But in our case we want to assess this relationship in accommodation establishments with quality certification .
\end{abstract}

The Industrial Safety studies analyzing this accident causes (why they happen), their sources (committed activities), their agents (participants work means), its type (how the events occur or develop), all in order to develop prevention.

In our case, as accidents happen because people commit wrongful acts or because the equipment, tools, machinery or workplaces are not in proper conditions, the preventive point of view we analyze through the incidence of workplace accidents hotel subsector to obtain the $Q$ tourist quality, since it combines system requirements and producto. The crash occurs because there is a risk, so that adequate control of it would avoid despite individual factors.

Absenteeism or absence from work was taken into account first by Dubois in 1977, as he realized the time lost in the nineteenth century due to the long working hours in Lasque included the holidays.

Motivation and job satisfaction were the elements that have been most important in the phenomenon of social psychology, analyzed by Nicholson, Brown and Chadwick Jones. In general, rate the absence from work as: 
An individual act in which you can choose alternate activities and a voluntary act that involves individual and group resistance with respect to an inflexible system.

Key issues that arise we are:

- What organizational structure should have the company to meet its targets for the prevention of occupational risks?

- How they can achieve their objectives in terms of risk prevention mean?

- Are the current organizations adequately protecting your workers?

- What is the relationship between accidents and Q quality certification ?

KEY WORDS: Accidents at work - Properties - Hotels - Q quality - Competitive Advantage.

\section{INTRODUCCIÓN}

Los accidentes de trabajo constituyen un problema importante a nivel mundial. Se calcula que cada año 2,34 millones de personas mueren de accidentes o enfermedades relacionados con el trabajo. De todas ellas, la gran mayoría - alrededor de 2,02 millones - fallecen a causa de una de las muchas enfermedades profesionales que existen. Además, la Organización Internacional del Trabajo calcula que cada año se producen 160 millones de casos de enfermedades no mortales relacionadas con el trabajo (OIT, 2013).

El turismo es un sector que opera en un entorno cada vez más exigente y más competitivo, lo que le lleva a velar por ofrecer servicios de calidad. Con esta realidad, y dado que hablamos de servicios, resulta fundamental cuidar los recursos humanos, el capital humano, puesto que las personas son el activo fundamental de una empresa turística y como tal debe ponerse en valor desarrollando su formación, informándolos, motivándolos, etc.

No obstante, cuando se habla de Recursos Humanos y Calidad de Servicios Prestados a menudo suele olvidarse que también es necesario para ello garantizar un ambiente de trabajo correcto y saludable. Todo ello repercutirá también positivamente en la buena marcha de la organización y, por ende, en la consecución de beneficios.

El bienestar del trabajador puede ser estudiado desde diversos prismas. Si nos quedamos en el estudio del bienestar del trabajador correlacionándolo exclusivamente con el salario, con el sueldo, pecaríamos de reduccionismo. Así, cuestiones como por ejemplo, la salud laboral, el bienestar laboral, el clima organizacional son factores que inciden claramente en el bienestar global del trabajador. Es necesario, por tanto, no solo tener en cuenta la faceta de bienestar del trabajador sino de considerarla desde una óptica amplia.

A principios de nuestro siglo comienzan a ser conocidos los accidentes de trabajo voluntarios en contextos de actividad con un grado de riesgo considerable para tener así una vía de escape y poder continuar con el puesto de trabajo. A estos accidentes 
junto con la situación anterior, se denominaba absentismo.

Simon, en 1978, opinaba que esta situación era una forma de resistencia de los campesinos hacia la forma de producción capitalista. Dubois, en una perspectiva más actual, sitúa el comportamiento absentista como una forma de acción obrera que se manifiesta para expresar el rechazo hacia la situación laboral o bien para poder obtener la remuneración sin trabajar y emplear el tiempo en otras actividades, como pasar el fin de semana completo con la familia, ir de vacaciones, realizar otros trabajos para tener así un sueldo extra o evitar impuestos. Mayo y un grupo investigador de Harvard hicieron un estudio en 1977 en tres compañías de una industria para estudiar el ausentismo y se descubrió que la influencia del grupo informal de trabajo en el individuo constituía un elemento clave para la determinación de la producción y de la presencia o ausencia laboral.

El precio tan elevado que supone la siniestralidad laboral para los países, las empresas y las personas, ha llevado a analizar la forma de reducirlo. Es preciso, por lo tanto, realizar estudios que permitan entender en toda su dimensión por qué se producen los accidentes de trabajo y las enfermedades profesionales así como los caminos o actuaciones para prevenirlos.

Así, el objetivo de este trabajo de investigación es dar a conocer, a través de su evolución en el periodo 2008-2011, la relación existente entre el número de establecimientos hoteleros a los que se les ha concedido la $Q$ de calidad y el número de accidentes laborales ocurridos en Andalucía en este subsector.

\section{OBJETIVOS}

Análisis descriptivo de la siniestralidad hotelera en relación a la implantación de un sistema de calidad.

$\checkmark$ Se pretende conseguir un programa de prevención que sea suficientemente atrayente, valorando su efectividad en la prevención de riesgos laborales.

$\checkmark$ Se valorará previamente la eficacia de diversos métodos de prevención empleados en experiencias realizadas por organizaciones del sector hotelero en determinados lugares afectados, uno de los indicadores de dicha eficacia será el absentismo laboral. Este análisis de siniestralidad hotelera se realizará por segmentos. Con ello se contestará a la pregunta de si las organizaciones actuales están protegiendo adecuadamente a sus trabajadores.

$\checkmark$ Nos centraremos en el problema que supone definir el fenómeno del absentismo laboral, ya que éste lleva aparejado una serie de comportamientos sin una relación aparente entre ellos. Hasta ahora no existen teorías que abarquen todas las interpretaciones que se han elaborado sobre el tema.

El programa de prevención que se pretende diseñar partirá de valorar los puntos débiles que resulten de este análisis previo y de propuestas de mejora. 


\section{METODOLOGÍA}

A fin de lograr el objetivo marcado se ha seguido una metodología descriptiva tomando como fuente de información secundaria la proporcionada por el propio Instituto para la Calidad Turística Española y el Instituto Nacional de Estadística.

Esta información de tipo longitudinal comprende el periodo 2008-2011, a la cual se le han calculado las variaciones porcentuales y la correlación lineal entre ambas variables, número de accidentes de trabajo y número de establecimientos hoteleros con Q de calidad.

\section{RESULTADOS}

La visión longitudinal de las variables número de accidentes de trabajo y número de establecimientos hoteleros que han obtenido la $Q$ de calidad nos permite observar cómo ha evolucionado el fenómeno objeto de estudio en este período de tiempo comprendido entre 2008 y 2011.

Es interesante destacar la disminución anual en el número de accidentes de trabajo y el incremento anual progresivo de los establecimientos hoteleros que han obtenido este reconocimiento.

Tabla 1. Accidentes de trabajo y establecimientos con Q de calidad

\begin{tabular}{|l|l|l|l|l|}
\hline & 2008 & 2009 & 2010 & 2011 \\
\hline Accidentes de trabajo & 9.998 & 8.599 & 8.311 & 7.961 \\
\hline Establecimientos con Q calidad & 12 & 21 & 26 & 31 \\
\hline
\end{tabular}

Fuente: INE, 2013 e Instituto para la Calidad Turística Española, 2013.

A partir de estas variables descriptivas calculamos sus variaciones porcentuales, es decir, la variación porcentual, a través del tiempo, de las cifras de accidentes de trabajo y de los establecimientos con $\mathrm{Q}$ de calidad. 
Tabla 2. Variación porcentual acontecida

\begin{tabular}{|l|l|l|l|}
\hline & 2009 & 2010 & 2011 \\
\hline Accidentes de trabajo & $-13,996$ & $-3,347$ & $-4,206$ \\
\hline Establecimientos con Q calidad & 75 & 23,809 & 19,231 \\
\hline
\end{tabular}

Fuente: Elaboración propia.

La variación porcentual para el número de accidentes de trabajo negativa, significa que la variable ha disminuido en valor. Caso contrario ocurre con los establecimientos con $\mathrm{Q}$ de calidad para los que la variación resultó positiva $\mathrm{y}$, por ende, dicha variable ha aumentado en valor.

A continuación, observamos lo anteriormente expuesto gráficamente.

Figura 1. Variación porcentual

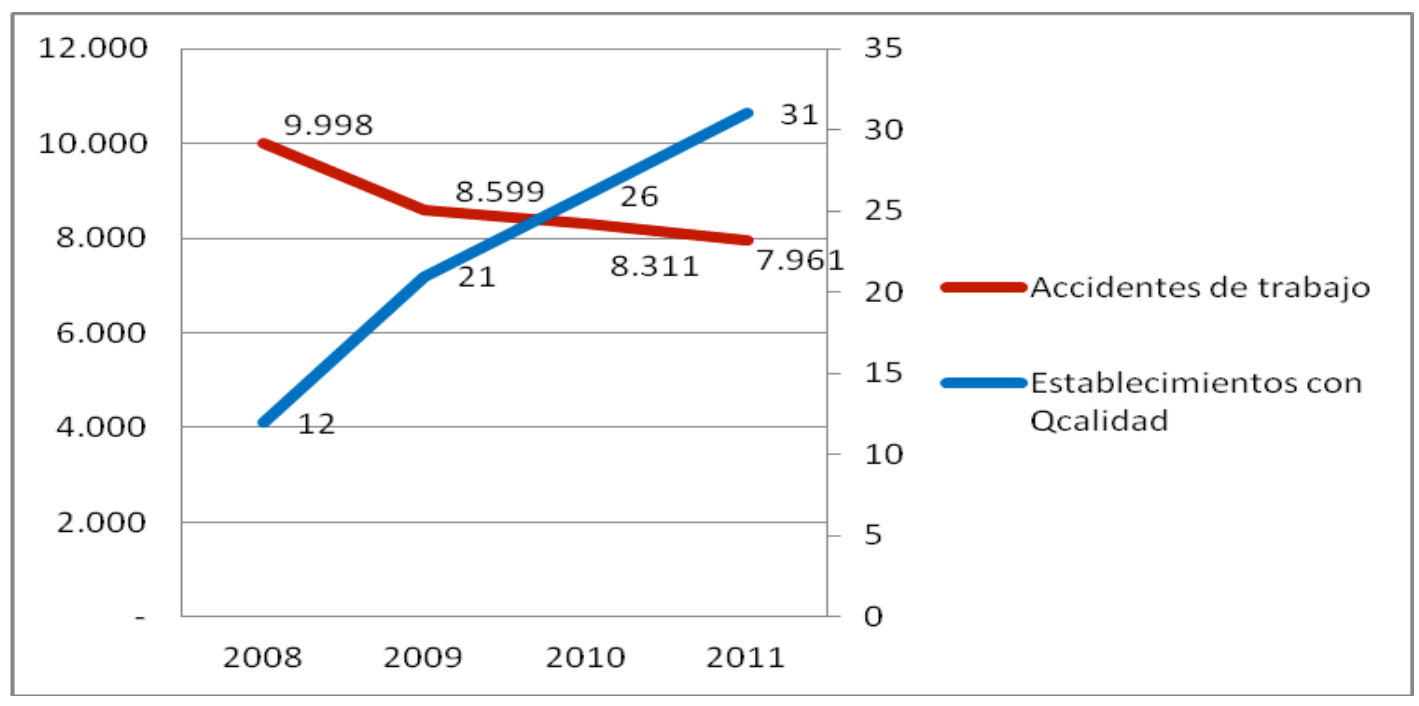

Fuente: Elaboración propia.

Detallamos que, al pasar de los años, el número de accidentes de trabajo se ha reducido conforme aumenta el número de establecimientos con $\mathrm{Q}$ de calidad.

No obstante, para confirmar estadísticamente lo anterior, efectuamos un gráfico de dispersión. Este gráfico muestra en un eje cartesiano la relación que existe entre dos variables; en el presente estudio las variables serán establecimientos con $Q$ de calidad y número de accidentes de trabajo. La relación viene expresada por el grado de correlación entre dichas variables, es decir, muestra si el incremento o decremento de los valores de la variable independiente (establecimientos con $Q$ de calidad) altera en cierta medida los valores de la variable dependiente (número de accidentes laborales). 


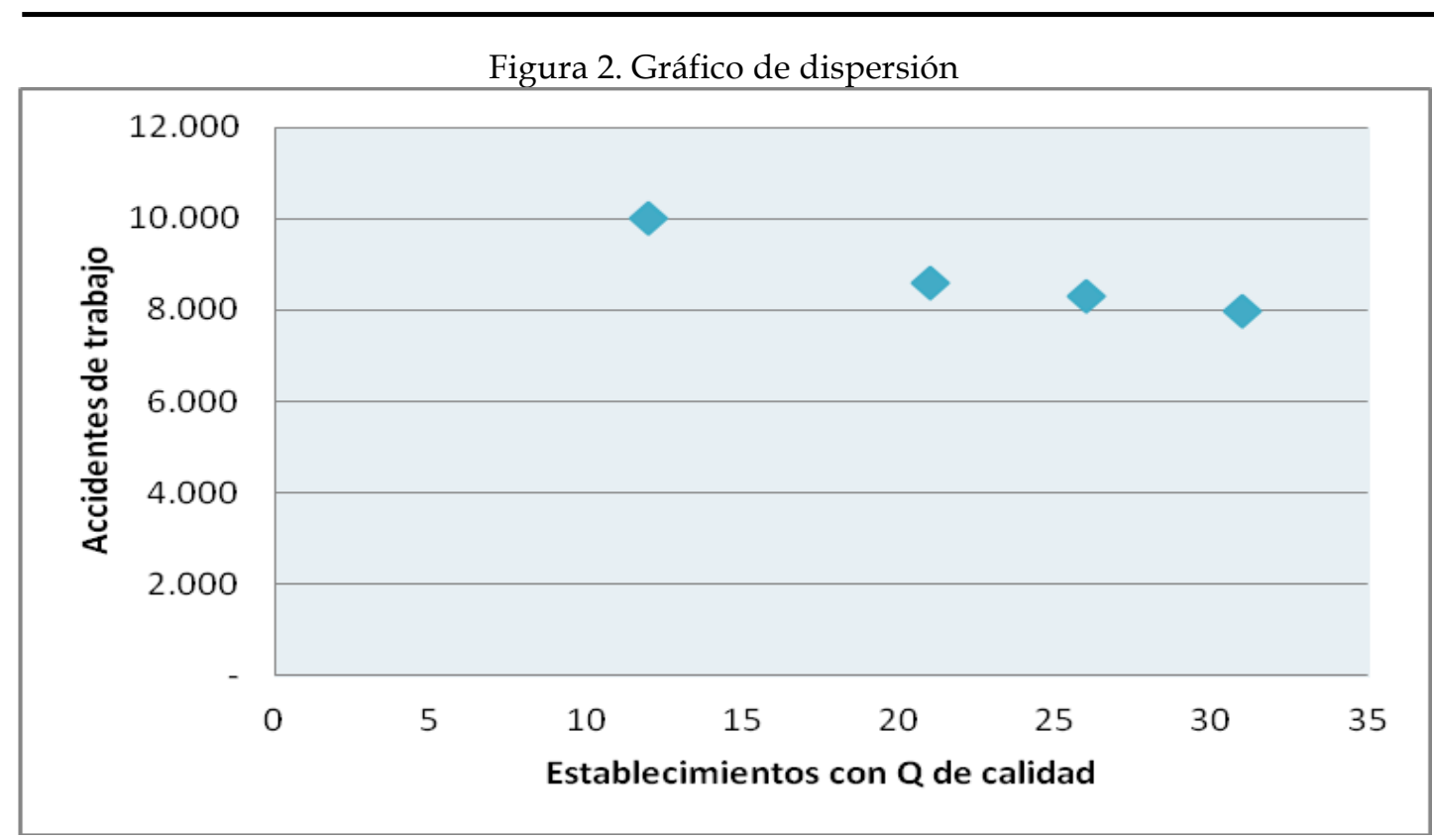

Fuente: Elaboración propia.

El tipo de correlación se observa en la nube de puntos del gráfico anterior. Podemos decir que existe una relación lineal negativa, ya que al aumentar los valores de la variable independiente disminuyen los valores de la variable dependiente. En otras palabras, con la presencia de más establecimientos con $Q$ de calidad, ocurren menos accidentes laborales.

Del mismo modo, realizamos el estudio anterior pero esta vez designando a la variable dependiente por el índice de accidentes mortales. En la tabla observamos los datos para el periodo de tiempo comprendido entre los años 2008 y 2011.

Tabla 3. Índices accidentes mortales y establecimientos con $Q$ de calidad

\begin{tabular}{|l|l|l|l|l|}
\hline & 2008 & 2009 & 2010 & 2011 \\
\hline Índice accidentes mortales & 0,83 & 0,87 & 0,86 & 0,85 \\
\hline Establecimientos con Q calidad & 12 & 21 & 26 & 31 \\
\hline
\end{tabular}

Fuente: Elaboración propia.

Calculando las variaciones porcentuales a este periodo obtenemos los datos de la siguiente tabla.

Tabla 4. Variación porcentual

\begin{tabular}{|l|l|l|l|}
\hline & 2009 & 2010 & 2011 \\
\hline Índice accidentes mortales & 5,585 & $-1,674$ & $-0,408$ \\
\hline Establecimientos con Q calidad & 75 & 23,809 & 19,230 \\
\hline
\end{tabular}

Fuente: Elaboración propia. 
Entre los años 2008 y 2009 aumentaron los accidentes mortales, pero a partir del año 2009 disminuyeron. Asimismo, como se determinó en el apartado anterior, los establecimientos con $Q$ de calidad aumentaron con el pasar de los años. En el gráfico se aprecia visualmente la representación de estas variables.

Figura 3. Establecimientos con $\mathrm{Q}$ de calidad y accidentes mortales

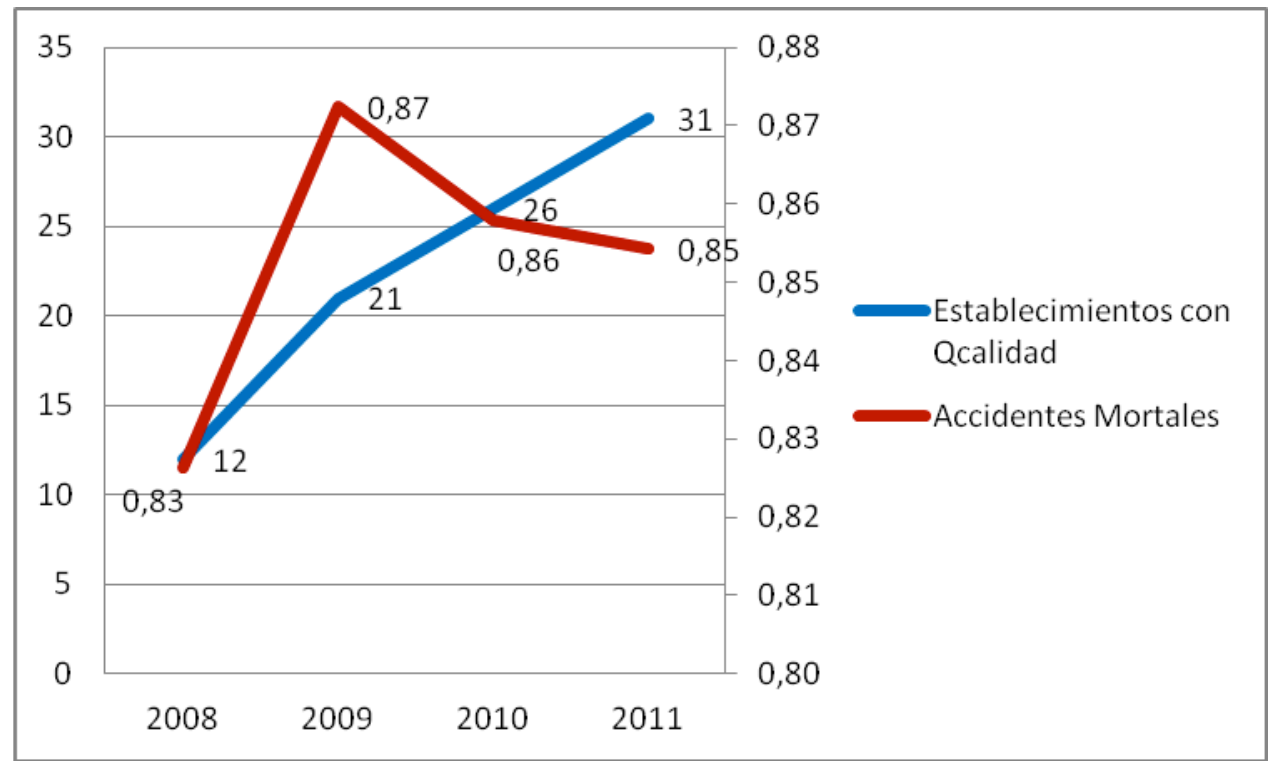

Fuente: Elaboración propia.

Del año 2008 al año 2009 hubo un incremento en el índice de accidentes mortales pero luego disminuyó hasta el año 2011. Por tanto, a partir del año 2009, se observa que pueden estar correlacionados los establecimientos con $Q$ de calidad con el índice de accidentes mortales, lo cual se analizará con el diagrama de dispersión en la siguiente figura. 
Figura 4. Establecimientos con Q de calidad e índices accidentes mortales

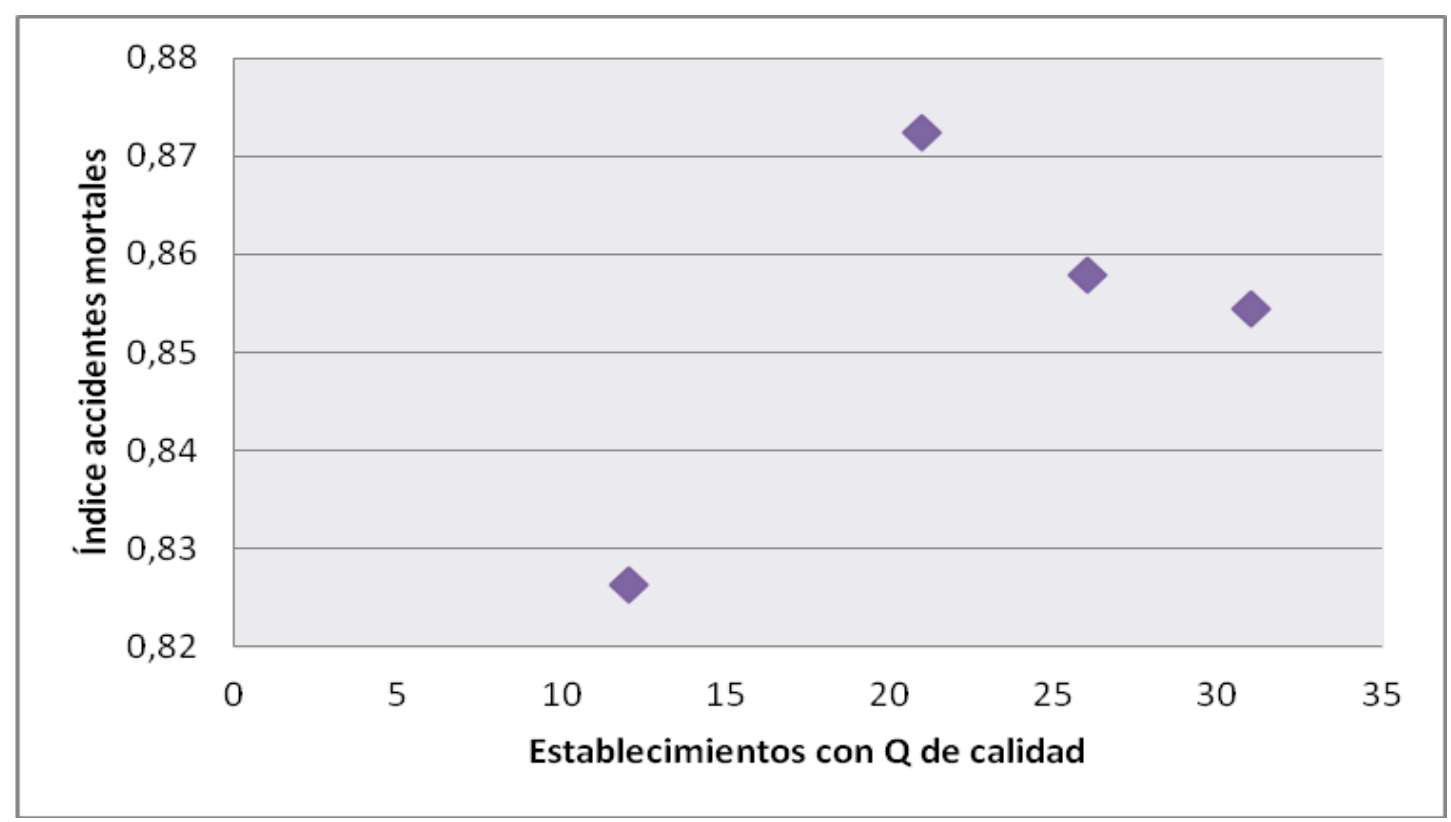

Fuente: Elaboración propia.

Como era de esperar, a partir del año 2009 hay una correlación negativa y, por ende, a mayor número de establecimientos con $Q$ de calidad menor será el índice de accidentes mortales.

\section{DISCUSIÓN}

Después de la investigación realizada en el presente estudio se puede hacer constar que es necesaria la creación de unas medidas especializadas en este sector, usándolas principalmente para potenciar y garantizar la prevención de riesgos laborales de los trabajadores del sector hotelero. Así como la importancia de la implantación de sistemas de certificación de calidad.

Los accidentes laborales son una lacra a nivel mundial. De ahí la necesidad de analizar cómo prevenirlos. Nuestro objetivo se ha centrado en analizar, a través de su evolución en el periodo 2008-2011, la relación existente entre el número de establecimientos hoteleros a los que se les ha concedido la $Q$ de calidad y el número de accidentes laborales ocurridos en Andalucía en este subsector.

Podemos concluir que existe una relación lineal negativa entre los accidentes laborales y el número de establecimientos con $Q$ de calidad, ya que al aumentar los valores de la variable independiente disminuyen los valores de la variable dependiente. En otras palabras, con la presencia de más establecimientos con $Q$ de calidad, ocurren menos accidentes laborales.

Por otro lado, a partir del año 2009 hay una correlación negativa entre el índice de 
accidentes mortales (que va disminuyendo) y el número de establecimientos con $\mathrm{Q}$ de calidad (que va en aumento).

La validez de este estudio se limita al periodo analizado que, aunque es interesante su análisis longitudinal, no nos permite una extrapolación de los resultados. Por ello, las futuras líneas de investigación deben ir encaminadas a corroborar las conclusiones obtenidas tanto en el ámbito analizado como a nivel nacional, internacional o global, máxime cuando en 2012 el número de empresas certificadas con Q de ICTE se ha reducido, en parte debido a la crisis actual, pues la certificación supone un coste y las subvenciones para ello han disminuido.

\section{REFERENCIAS}

Giráldez, P., Martín, M. A. (2004). La rentabilidad en el sector hotelero: eficiencia y concentración. Universidad de Sevilla, Secretariado de publicaciones. Sevilla.

Martín, I. (2014): “La calidad en las empresas turísticas” en Aguiló, E. y González, A. (Coordinadores) Dinámica sectorial y estrategias empresariales, Actividad Turística Española en 2012. Madrid. pp. 101-109.

Organización Internacional del Trabajo (OIT) (2001). Memoria del director general: actividades de la O.I.T. 1998-1999. Madrid.

Organización Internacional del Trabajo (OIT) (2013). The prevention of occupational Diseases. Ginebra. Disponible en www.ilo.org/wcmsp5/groups/public/---ed_protect/--protrav/---safework/documents/publication/wcms_208226.pdf (Consultado 12/II/2014).

Tous Pallarés, J. (2009). El comportamiento absentista y sus repercusiones en la calidad de vida y la calidad de servicio en el sector hotelero. 